\title{
MÉTOdOS ESTATÍSTICOS PARA AGRUPAMENTO NÃO HIERÁRQUICOS DE DADOS
}

E.Acarini'

G.Amaral

RESUMO

Os métodos estatísticos de análise de agrupamento (Cluster Analysis) possibilitam estudar um conjunto de dados subdividindo-o em diversos grupos, com características internas semelhantes, e distintas entre si. Os métodos K-médias e Isodata procuram estabelecer tais relações sem qualquer base hierárquica influindo no processo de formação de grupos. Isto possibilita uma grande mobilidade para as unidades de dados analisadas e a conseqüente formação de grupos com relações internas mais próximas. O método K-médias utiliza-se de distâncias euclideanas entre as médias das variáveis de um grupo, em relação a cada unidade do conjunto de dados, procurando estabelecer $\mathrm{K}$ grupos com as menores distâncias internas possiveis. O método Isodata opera com valores paramétricos, permitindo a individualização de um número qualquer de grupos.

O presente trabalho procura apresentar dois programas desenvolvidos em FORTRAN 77, Centro de Processamento de Dados do Instituto de Geociências da Universidade de São Paulo, e uma breve análise dos resultados obtidos por programas quando aplicados a um exemplo real.

\section{ABSTRACT}

${ }^{1}$ Bolsista de Iniciação Cientifica (FAPESP), Instituto de Geociências/USP, São Paulo.

${ }^{2}$ Departamento de Paleontologia e Estratigrafia, Instituto de Geociências/USP, Săo Paulo. 
A cluster is usually thought to be a subset of data points which are highly similar or associated and relatively unassociated with data points outside the subset. Cluster Analysis makes it possible to study data sets and subdivide them into several distinct clusters with similar internal characteristics. The K-means and ISODATA algorithms can establish this relationship, without any hierarchical base influencing in the cluster formation process. This gives great mobility to the data units analyzed and facilitates the consequent formation of clusters with the closest internal relationships. The K-means algorithm uses Euclidean distances between the means of the variables of a cluster with respect to each unit of the data set and estabilishes $\mathrm{K}$ clusters for the smallest interval distance possible. In the ISODATA technique (which operates in the same way) each pattern is put into cluster for which the squared distance between it and the cluster mean is smallest. The new clusters means are then computed and the whole procedure repeated.

\section{INTRODUÇÃO}

Os métodos estatísticos de análise de agrupamento (Cluster Analysis) surgiram da necessidade de classificar, reunir e separar os objetos em estudo de acordo com suas características mensuráveis.

Uma aplicação, que se tem mostrado bastante útil em geologia, é a subdivisão em grupos de amostras analisadas quimicamente. As informações obtidas a partir deste agrupamento podem permitir relacionar cada grupo a unidades litológicas com características geoquímicas próprias.

\section{FUNDAMENTOS TEÓRICOS}

Inicialmente serão explicados os conceitos básicos envolvidos nos programas.

Uma população é um conjunto de objetos ou indivíduos, finitos ou infinitos, que constitui o conjunto universo. Para se analisar uma população não são necessários dados de toda população, mas apenas de uma pequena parte, chamada amostra.

Os métodos estatísticos permitem o estudo de uma população (ou amostra), cuja distribuição apresenta certas características, conhecidas como parâmetros, tais como: o ponto médio, as medidas de simetria da distribuição e as medidas que indicam o valor da dispersão. 
Os programas utilizam como um dos parâmetros a média aritmética, que é definida como a soma de todas as observações dividida pelo número de observações.

$$
\bar{x}=\frac{\sum_{i=1} x_{i}}{N}
$$

Um outro parâmetro é a variância, que mede a dispersão dos elementos de uma amostra em relação a média

$$
s^{2}=\frac{\sum_{i=1}^{N}\left(x_{i}-\bar{x}\right)^{2}}{N}
$$

$$
\begin{aligned}
& X=\text { média } \\
& N=\text { números de elementos }
\end{aligned}
$$

e o desvio padrão, que é a raiz quadrada da variância.

$\mathrm{Na}$ natureza é comum serem encontrados conjuntos de populações com distribuição estatística normal (ou distribuição de Gauss) definida pela equação

$$
Y=\frac{1}{\sigma 2 \Pi} e-1 / 2(x-\mu)^{2} / \sigma^{2}
$$

porém outros tipos de distribuição (binominal, Poisson, etc) também podem ser encontradas.

As populações podem, no entanto, apresentar dificuldades quando se procura separá-las. Para tal, utiliza-se a análise de variância, que proporciona a caracterização das diferenças existentes entre as populações do conjunto.

A Figura 1 assume que a variável analisada possui distribuição estatística normal, com a mesma variância em cada população. Nota-se que todas as populações são centradas em pontos diferentes e possuem distribuição contínua.

Novas técnicas, reunidas sob a designação de análises multivariantes, procuram estudar grupos diferentes de populações em um espaço multidimensional. A diferença entre a análise de variância e a análise multivariante é que aquela possui uma única variável dependente e esta possui 
um vetor de variáveis. A população agora não está mais centrada em torno de um grupo, mas em torno de um vetor de variáveis (Fig. 2).

Os parâmetros utilizados pela análise multivariante são extrapolações dos parâmetros da análise de variância (ANOVA).

Os métodos K-médias e ISODATA constroem grupos com técnicas da análise multivariante. Estes dois métodos são hierárquicos de agrupamentos, $\theta$ são assim denominados porque agrupam unidades de dados em vários grupos, que podem ser especificados a priori (Kmédias), ou determinados como parte do método de agrupamento (ISODATA). Diferem dos métodos hierárquicos por não fornecerem uma "árvore" de classificação.

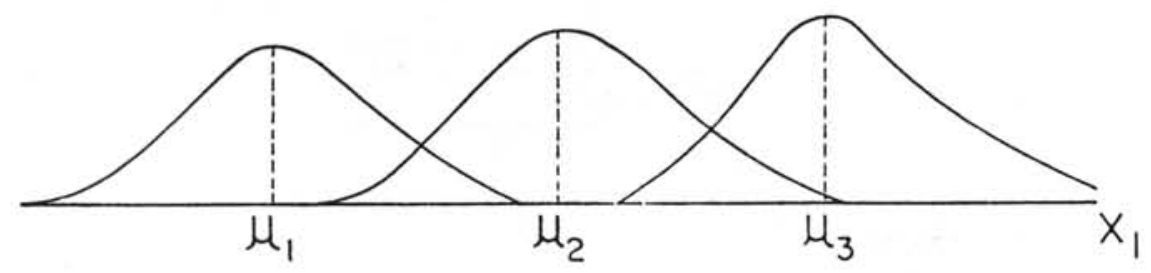

Figura 1 - Populaçōes diferenciadas pela análise de variância.

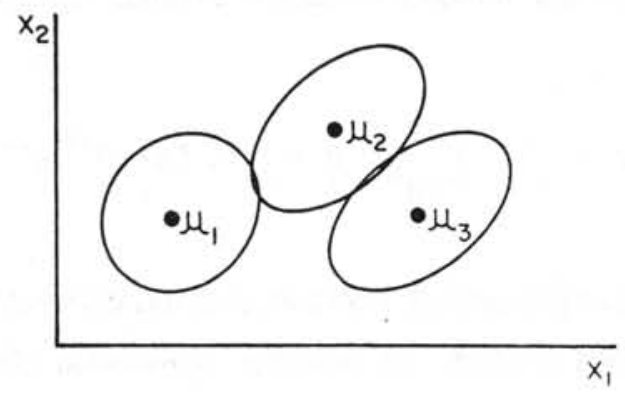

Figura 2 - Populaçōes distinguidas pela análise multivariante (apenas duas variáveis).

\section{ESTRUTURAS DOS DADOS}

A estrutura dos dados é constituída de um número de casos (ou amostras), e cada caso é constituído de uma série de variáveis (por exemplo: as porcentagens em óxidos de uma amostra de rocha). 


\section{NORMALIZAÇÃO E PADRONIZAÇÃO}

Muitas vezes é necessário destacar variáveis que apresentem valores extremamente pequenos e comparátos a variáveis como valores mais expressivos. Um exemplo ilustrativo é apresentado na Tabela 1. Trata-se de uma série de análises químicas de rochas carbonáticas do Permiano da Bacia do Maranhão (COIMBRA, 1983). Os resultados analfticos estão em ppm e evidenciam uma grande diferença entre as variáveis. Destacam-se de um lado os elementos traços, como o $\mathrm{Ni}, \mathrm{Zr}$, Sr e de outro os elementos maiores, como o $\mathrm{Ca}, \mathrm{Al}, \mathrm{Mg}$.

Para comparar os resultados destas análises seria necessário normalizar, criar um critério para equilibrar o peso das variáveis. Um modo de se fazer isto é estabelecer o valor 100 para o maior valor da variável e o valor 0 (zero) para o menor valor, e interpolar os demais valores dentro deste intervalo. Outro modo é o da padronização estatistica, onde cada valor é subtraído de sua média e dividido pelo desvio padrão, resultando numa nova variável com média zero e variância unitária.

\section{O MÉTODO K-MÉDIAS}

O objetivo deste algoritmo é pesquisar a melhor partição de dados, em que cada grupo formado abrangerá os casos mais próximos em relação a sua média. Este método foi desenvolvido inicialmente por MacQUEEM (1967) e modificado posteriomente por ANDERBERG (1973).

\section{Considerações iniciais}

O I-ésimo caso da J-ésima variável possui o valor $A(I, J)\{I=1 \ldots M, J=1 \ldots N\}$. Vamos supor que exista preliminarmente uma partição dos $M$ casos em $K$ agrupamentos, $P(M, K)$. Cada grupo é representado pela média das variáveis dos casos que o compõe, MÉDIA (L,J). Local (I) designa o grupo ao qual pertence o caso I. O número de casos no grupo $L$ é $N(L)$ e a distância euclideana entre o Lésimo grupo e o lésimo caso é dada por:

$$
D(I, K)=\sqrt{\sum_{J=1}^{N}(A(I, J)-\operatorname{MidIA}(L, J))^{2}}
$$


O erro cometido por um determinado particionamento do conjunto de dados é dado por:

$$
e=\sum_{I=1}^{M} D(I, \operatorname{LOCAL}(I))^{2}
$$

\section{PROCEDIMENTOS}

1 - calcular a média dos grupos e o erro da partição inicial;

2 - calcula-se, para cada caso I, a distância entre este caso e os grupos formados nesta partição;

3 - caso I, pertencente a grupo L(I), esteja mais próximo do grupo L, mude I do grupo $L(I)$ para o grupo L Atualize a média e corrija o erro;

4 - repita o passo 2 até $I=M$;

5 - se não ocorrer mais nenhum movimento de casos de um grupo para o outro, pare; caso contrário, volte ao passo 2.

\section{O MÉTODO ISODATA}

Dado um conjunto inicial de dados, o Isodata reparte este conjunto em grupos distintos, com base em valores paramétricos, buscando a melhor partição através da separação, combinação e eliminação dos grupos. Este método fol desenvolvido por BAL \& HALL (1965).

\section{Considerações iniciais}

Inicialmente deve-se fornecer os parâmetros, que servirão de base para a separação, combinação e eliminação dos grupos. Estes parâmetros são: o desvio padrão máximo, DPMAX, que é o parâmetro acima do qual um grupo se separará; a distância mínima, DEMIN, abaixo da qual grupos distintos devem combinar-se; o número mínimo de pontos que um grupo deve conter, NMIN, abaixo do qual o grupo é eliminado. Também devem ser fornecidos o número de iterações a ser realizado, NITER, e a seqüência de passos a serem executados. 
Tabela 1 - Análises químicas das rochas carbonáticas da Bacia do Maranhão (com algumas descrições litológicas).

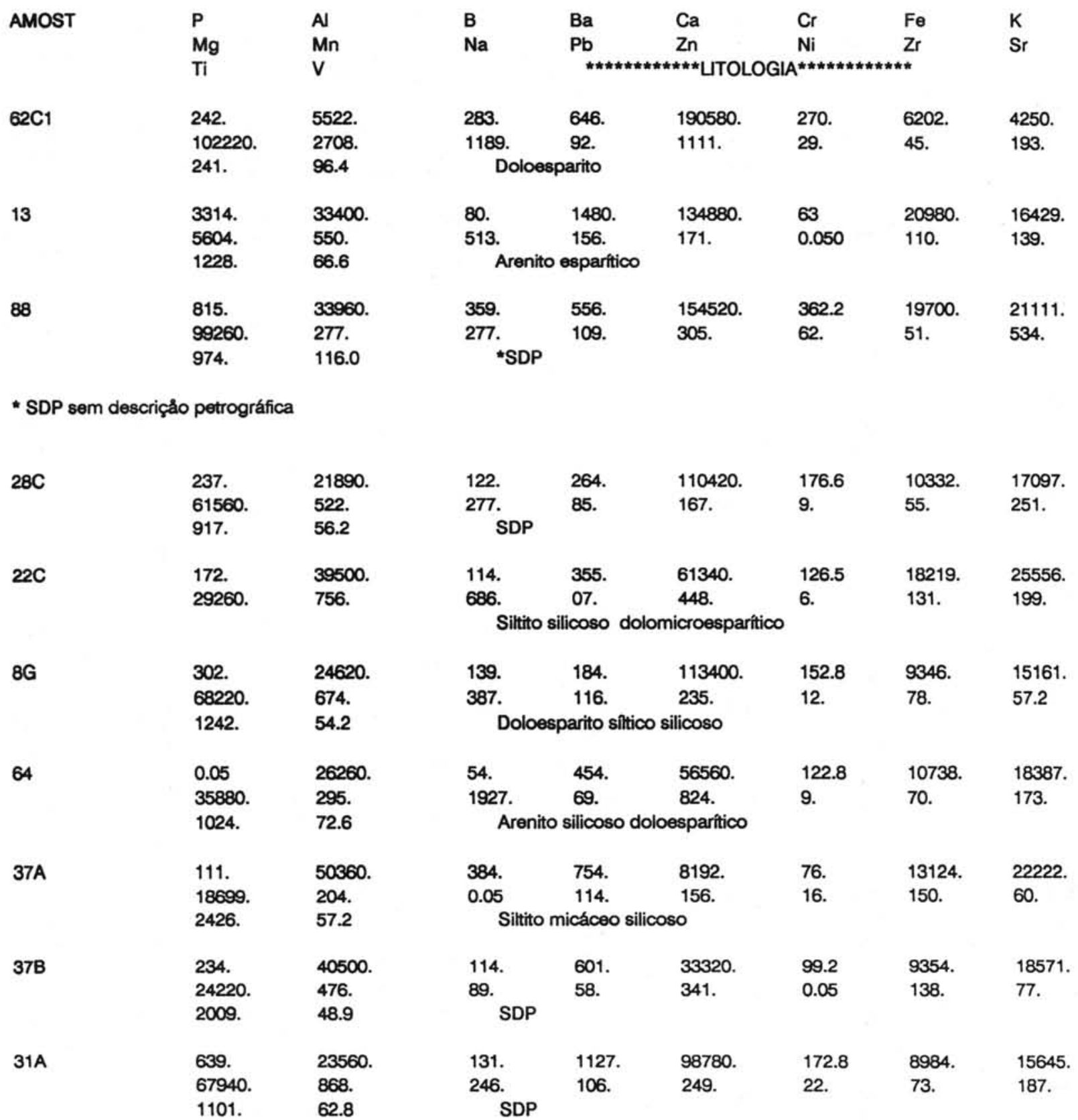




\begin{tabular}{|c|c|c|c|c|c|c|c|c|}
\hline $89 \mathrm{~A}$ & $\begin{array}{l}3398 . \\
79780 .\end{array}$ & $\begin{array}{l}7796 . \\
1728 .\end{array}$ & $\begin{array}{l}122 . \\
1550 .\end{array}$ & $\begin{array}{l}219 . \\
96 .\end{array}$ & $\begin{array}{l}141720 \\
147 .\end{array}$ & $\begin{array}{l}196.8 \\
0.05\end{array}$ & $\begin{array}{l}5998 . \\
41 .\end{array}$ & $\begin{array}{l}4786 . \\
883 .\end{array}$ \\
\hline & 245. & 61.8 & \multicolumn{3}{|c|}{ Doloesparico arenoso } & & & \\
\hline \multirow[t]{2}{*}{ 31D } & $\begin{array}{l}307 . \\
80180 .\end{array}$ & $\begin{array}{l}25140 . \\
855 .\end{array}$ & $\begin{array}{l}177 . \\
214 .\end{array}$ & $\begin{array}{l}300 . \\
115 .\end{array}$ & $\begin{array}{l}125620 . \\
126 .\end{array}$ & $\begin{array}{l}230.2 \\
42 .\end{array}$ & $\begin{array}{l}9910 . \\
83 .\end{array}$ & $\begin{array}{l}4143 . \\
74 .\end{array}$ \\
\hline & 1151. & 63.6 & \multicolumn{3}{|c|}{ Doloesparito sítico silicoso } & & & \\
\hline \multirow[t]{2}{*}{$\approx \mathrm{C} 2$} & $\begin{array}{l}0.05 \\
24000 .\end{array}$ & $\begin{array}{l}1595 . \\
340 .\end{array}$ & $\begin{array}{l}88 . \\
2432 .\end{array}$ & $\begin{array}{l}256 . \\
43 .\end{array}$ & $\begin{array}{l}78240 . \\
232 .\end{array}$ & $\begin{array}{l}73.2 \\
0.05\end{array}$ & $\begin{array}{l}3374 . \\
31 .\end{array}$ & 9565. \\
\hline & 346. & 60.4 & \multicolumn{3}{|c|}{ Silexito arenoso doloesparitico } & & & \\
\hline \multirow[t]{2}{*}{$30 \mathrm{~B}$} & $\begin{array}{l}163 . \\
20640 .\end{array}$ & $\begin{array}{l}10088 . \\
329 .\end{array}$ & $\begin{array}{l}351 . \\
0.05\end{array}$ & $\begin{array}{l}107 . \\
70 .\end{array}$ & $\begin{array}{l}120500 . \\
170 .\end{array}$ & $\begin{array}{l}80.8 \\
0.05\end{array}$ & $\begin{array}{l}4738 . \\
37 .\end{array}$ & $\begin{array}{l}6521 . \\
97 .\end{array}$ \\
\hline & 244. & 29.8 & \multicolumn{4}{|c|}{ Silexito arenoso esparítico magnesiano } & & \\
\hline \multirow[t]{2}{*}{$28 \mathrm{E}$} & 0.05 & 19966. & 55. & 157. & 84760. & 133.2 & 9730. & 11935. \\
\hline & $\begin{array}{l}55400 . \\
990 .\end{array}$ & $\begin{array}{l}518 . \\
49.2\end{array}$ & \multicolumn{2}{|c|}{ SDP } & 84. & 0.05 & & \\
\hline \multirow[t]{3}{*}{41} & 117. & 2024. & 46. & 950. & 366600. & 64.2 & 1046. & 33333. \\
\hline & 2700. & 675. & 0.05 & 30. & 50. & 0.05 & 5. & 170. \\
\hline & 97. & 3.4 & \multicolumn{2}{|c|}{ Esparito } & & & & \\
\hline \multirow[t]{2}{*}{$58 \mathrm{C}$} & 88. & 33440 . & 63. & 421. & 45040. & 117.8 & 4732. & 1000. \\
\hline & $\begin{array}{l}26420 . \\
1373 .\end{array}$ & $\begin{array}{l}235 . \\
116.4\end{array}$ & \multicolumn{2}{|c|}{ SDP } & 57. & 19. & 108. & 130. \\
\hline \multirow[t]{3}{*}{ 28B } & 77. & 25380. & 207. & 159. & 94460. & 176.8 & 12894. & 18871. \\
\hline & 55720. & 499. & 0.05 & 105. & 108. & 0.05 & 53. & 428. \\
\hline & 1034. & 57.4 & \multicolumn{2}{|c|}{ SDP } & & & & \\
\hline \multirow[t]{3}{*}{$58 \mathrm{~A}$} & 183. & 22240. & 88. & 283. & 93100. & 166.6 & 8206. & 13870. \\
\hline & 57700. & 464. & 717. & 77. & 81. & 17. & 65. & 191. \\
\hline & 1011. & 42.4 & \multicolumn{3}{|c|}{ Arenito doloesparítico silicoso } & & & \\
\hline \multirow[t]{3}{*}{ 62F } & 88. & 15558. & 131. & 361. & 141100. & 171.8 & 5056. & 9673. \\
\hline & 79160. & 1169. & 2542. & 94. & 180. & 0.05 & 57. & 133. \\
\hline & 649. & 65.6 & \multicolumn{3}{|c|}{ Doloesparito arenosiltoso } & & & \\
\hline \multirow[t]{2}{*}{90} & 292. & 16528. & 182. & 280. & 154720. & 228. & 17064. & 10645. \\
\hline & 735. & 84.2 & \multicolumn{3}{|c|}{ Doloesparito silicoso } & & & \\
\hline \multirow[t]{3}{*}{ 22B } & 2380. & 49760. & 207. & 464. & 46760. & 78. & 18622. & 24444. \\
\hline & 19384. & 50. & 183. & 121. & 123. & 17. & 130. & 120. \\
\hline & 2396. & 79.6 & \multicolumn{4}{|c|}{ Siltito silicoso dolomicroesparítico } & & \\
\hline \multirow[t]{3}{*}{$89 B$} & 314. & 6484. & 105. & 201. & 160280. & 174.4 & 7024. & 4143. \\
\hline & 92000. & 1780. & 937. & 55. & 102 & 9. & 38. & 846. \\
\hline & 221. & 46.2 & \multicolumn{2}{|c|}{ Doloesparito } & & & & \\
\hline \multirow[t]{2}{*}{$38 B$} & 0.05 & 54760. & 46. & 302. & 9798. & 35.8 & 20380. & 1000. \\
\hline & $\begin{array}{l}15686 . \\
1016 .\end{array}$ & $\begin{array}{l}301 . \\
71.4\end{array}$ & \multicolumn{2}{|c|}{ SDP } & 408. & 3. & 138. & 76. \\
\hline
\end{tabular}


$30 \mathrm{~A}$

$35 \mathrm{C}$

$38 A$

31B

62D

35D

$58 \mathrm{H}$

$58 B$

$28 \mathrm{~F}$

$35 \mathrm{~A}$

$22 \mathrm{C}$

79

$35 \mathrm{~B}$

$\begin{array}{ll}0.05 & 21380 . \\ 30160 . & 262 . \\ 950 . & 0.05\end{array}$

$75 . \quad 14264$.

$39800 . \quad 531$.

950.

\subsection{5}

41580.

572.

99.

11172.

490.

106.4

13. 211980.

7490 . 375.

$1459 . \quad 80.2$

$0.05 \quad 11172$.

$52620 . \quad 490$

621.

74.4

138.22160.

26580.223.

1156. 92.6

$28 . \quad 18000$.

6598. 1311.

1219. $\quad 102.8$

822.12516.

$67400 . \quad 606$.

583. $\quad 68.6$

$12 . \quad 5750$.

$62640 . \quad 327$.

344. $\quad 456.2$

22.15030.

43420 . 402 .

$648 . \quad 64$.

$0.05 \quad 11904$.

$5714 . \quad 1318$.

592. 89.80

$194 . \quad 28100$.

27900.1091.

$2158 . \quad 194.4$

$135 . \quad 15992$.

59880.449.

792. $\quad 76.2$

109.2398.

9722.11185

1087.

$\begin{array}{ll}46 . & 102 \\ \text { SDP } & \end{array}$

$129 . \quad 129$

4916.

168.

100840

563.

42560.

25.8

0.05

10122. 15161

34.

138.

\section{SDP}

144.

3752.

443. 284800 . 127.

83.

Esparito magnesiano

\begin{tabular}{|c|c|c|c|c|}
\hline $\begin{array}{l}122 . \\
3958 .\end{array}$ & $\begin{array}{l}301 . \\
146 .\end{array}$ & $\begin{array}{l}6520 . \\
122 .\end{array}$ & $\begin{array}{l}92 . \\
26 .\end{array}$ & $\begin{array}{l}9928 . \\
114 .\end{array}$ \\
\hline $\begin{array}{l}114 . \\
3028\end{array}$ & $\begin{array}{l}230 . \\
85 .\end{array}$ & $\begin{array}{l}80180 . \\
116 .\end{array}$ & $\begin{array}{l}144 . \\
9 .\end{array}$ & $\begin{array}{l}6100 . \\
63 .\end{array}$ \\
\hline
\end{tabular}

Silexito arenoso doloespartíico

$\begin{array}{llllll}80 . & 295 . & 47820 . & 97 . & 9118 . & 18871 . \\ 6112 . & 126 . & 85 . & 13 . & 91 . & 136 .\end{array}$

Arenito doloesparítico silicoso

$\begin{array}{llllll}207 . & 353 . & 210800 . & 42 . & 14716 . & 12742 . \\ 3140 . & 134 . & 105 . & 36 . & 67 . & 559 .\end{array}$

$\begin{array}{llllll}156 . & 258 . & 103460 . & 157 . & 5900 . & 9022 .\end{array}$

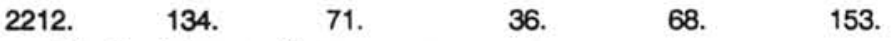

Silexito doloesparitico

$\begin{array}{llllll}359 . & 193 . & 123960 . & 218.0 & 2582 . & 1714 . \\ 1944 . & 74 . & 53 . & 26 . & 52 . & 274 .\end{array}$

Biodoloesparito silicoso

$\begin{array}{llllll}46 . & 84 . & 71980 . & 108 . & 7526 . & 8281 . \\ 2054 . & 116 . & 68 . & 29 . & 56 . & 51 .\end{array}$

\section{SDP}

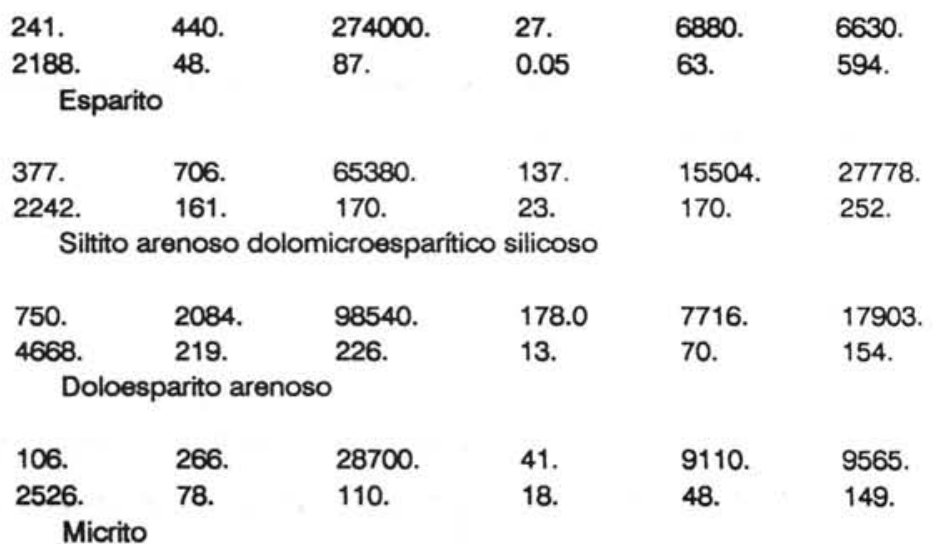




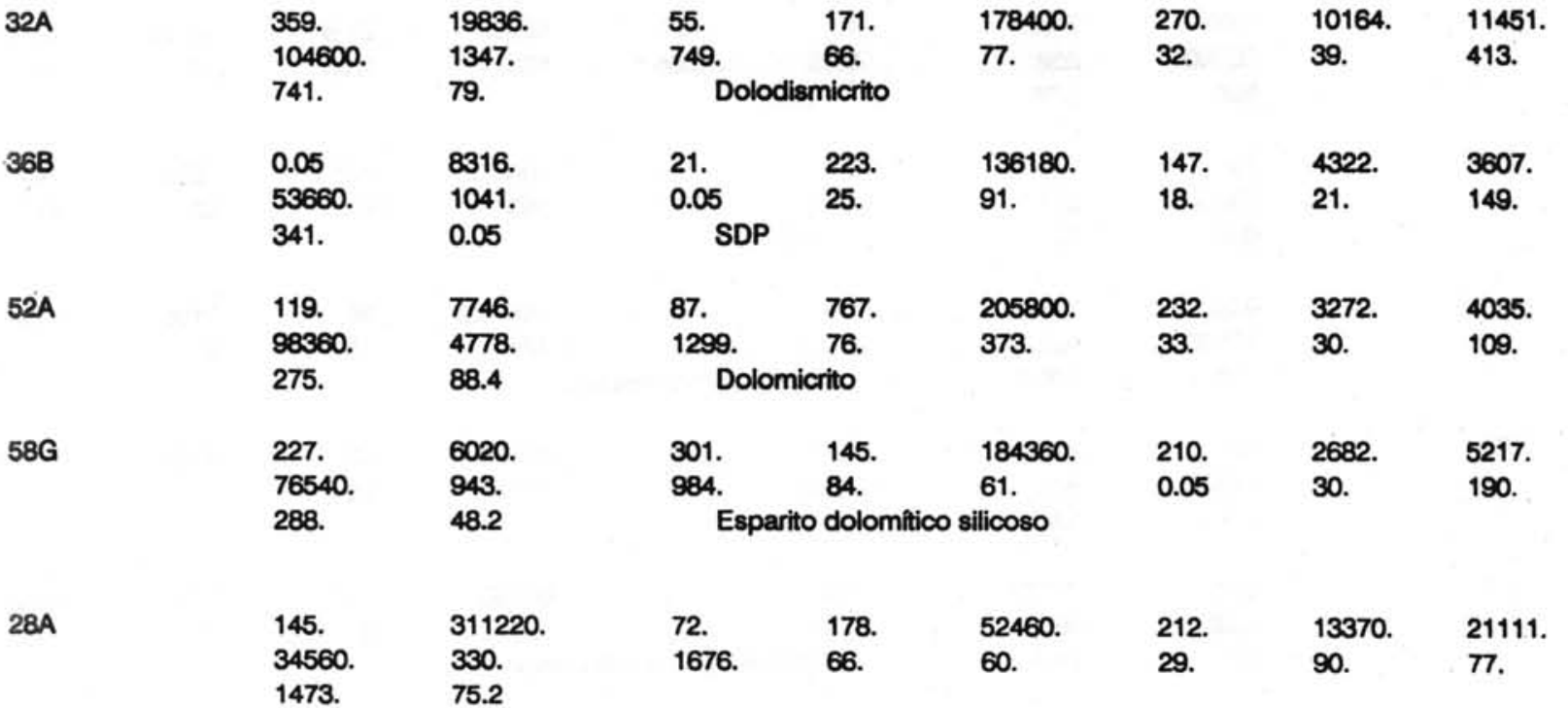

PROCEDIMENTOS

0 - inicializa (lê uma matriz de dados e os parâmetros, calcula a média e os desvios padrões iniciais);

1 - calcula a distância euclideana entre os grupos;

2 - muda a estrutura dos grupos;

2.1 - elimina - destrói grupos;

2.2 - separa se for uma iteração do tipo (1) e val para 3, senão continua;

2.3 - combina

3 - Se é a última iteração numa seqũência Separa(1)-combina(0), senão vai para a próxima iteração, para 1.

\section{EXEMPLO}

Como exemplo de aplicaçăo foram utilizados os dados de análises químicas de rochas carbonáticas do Permiano da Bacia do Maranhão (COIMBRA, 1983). Sobre estes dados foram 
aplicados os dois métodos de agrupamento discutidos anteriormente, com as varívels normalizadas e não normalizadas. Em seguida procurou-se indentificar os principais fatores $\theta$ variáveis que influenciaram no resultado.

Apresentam-se, na Tabela 1, os dados que representam resultados de and́lises químicas de rochas da Formaçăo Pedra de Fogo e Motuca.

\section{ANÁLISE DOS RESULTADOS}

\section{Varíaveis não padronizadas}

\section{K-médias}

Ao aplicarmos o K-médlas sobre estes dados foram realizadas várias sugestões de subdivisăo de grupos $(2,3,4,5,6,9,11)$. Todos os resultados mostraram grupos com relações internas em maior ou menor grau, dependendo unicamente do nivel de detalhe exigido. Quando é sugerida uma subdlvisão em apenas dois grupos, consegue-se separar as rochas carbonáticas com maior contribuiçăo de terrigenos. Detalhando-se melhor esta subdivisão obtém-se :

\section{AMOSTRAS}

GRUPO 1: 22A, 22B, 22Ç 28A, 30A, 37A, 37B, 38B, 58Ç 62D, 64

GRUPO 2: 38A

GRUPO 3:28B, 28Ç 28D, 28E, 28F, 28G, 30B, 31A, 31B, 31D, 36B, 58A, 58B, 58H, 62C2, 79

GRUPO 4: 32A, 52A, 58G, 62C1, 62F, 88, 89A, 89B, 90

GRUPO 5: 13, 35D

GRUPO 6: 35A, 35B, 35Ç 41

Estes 6 grupos foram obtidos com as variáveis não padronizadas. Isto implica que as variáveis com valores maiores influenciaram mais o resultado, ou seja, os valores de $\mathrm{Ca}, \mathrm{Mg}, \mathrm{Al}$, $\mathrm{Fe}$, e $\mathrm{K}$ foram os fatores determinantes na subdivisão dos grupos.

A Tabela 2 procura definir os teores, em termos relativos, e as características de cada grupo, em termos dos 5 elementos que mais influenciaram na subdivisão.

Do resultado (Tabela 2) e da descrição litológica das amostras (COIMBRA, 1983), podemos caracterizar cada grupo como segue : 
GRUPO 1: siltitos, arenitos e margas dolomfticas da Formaçăo Pedra de Fogo.

GRUPO 2: argilas com muito pouco carbonato da Formação Pedra de Fogo.

GRUPO 3: dolomitos, dolomitos arenosos e arenitos dolomiticos da Formação

Pedra de Fogo.

GRUPO 4: calcáreos dolomfticos da Formação Pedra de Fogo e Motuca.

GRUPO5: calcáreos calcfticos sfiticos da Formaçăo Motuca e Formação Pedra de

Fogo.

GRUPO 6 : calcáreos calcfiticos da Formação Motuca.

Isodata

O Isodata propicia resultados em funçăo da adoçăo de três parâmetros: desvio padrăo máximo que uma variável pode assumir dentro de um grupo, a distância mínima entre dois grupos e o número mínimo de pontos que um grupo deve conter. Para as variáveis não padronizadas é fácil notar que estas possuem uma ampla distribuição, o que implica em desvios padrões e distâncias com valores elevados.

O resultado obtido pelo Isodata, adotando-se um desvio padrão de 1100 , uma distância de 460 e um número mínimo de 2 unidades de dados para cada grupo, é:

Amostras ignoradas: $38 \mathrm{~A}, 41,58 \mathrm{C}$

\section{AMOSTRAS}

GRUPO 1: 22A, 22B, 22Ç 28A, 28B, 28Ç 28D, 28E, 28F, 28G, 30A, 31A, 31B, 36B, $37 \mathrm{~B}, 58 \mathrm{~A}, 58 \mathrm{~B}, 58 \mathrm{H}, 62 \mathrm{C} 2,62 \mathrm{D}, 64,79$

GRUPO 2: 35A, 35B, 35C

GRUPO 3: 13, 30B, 31D, 62F, 89A

GRUPO 4: 88, 89B, 90

GRUPO 5: 32A, 35D, 52A, 58G, 62C1

GRUPO 6: 37A, 38B

Como podemos observar pela Tabela 3, o Isodata deixa-se influenciar marcantemente pelas variáveis que possuem os maiores valores e em decorrência os maiores desvios padrões. Observa-se que a subdivisão dos grupos sofreu forte influência das variáveis $\mathrm{Ca}$ e $\mathrm{Mg}$ e, secundariamente, das variáveis Al, Fe e K. 
Tabela 2 - Valores Teores relativos à mediana para os grupos obtidos pelo programa K-médias.

\begin{tabular}{|c|c|c|c|c|c|c|}
\hline ELEM. & GRUPO 1 & GRUPO 2 & GRUPO 3 & GRUPO 4 & GRUPO 5 & GRUPO 6 \\
\hline Ca & $\begin{array}{l}\text { teores mais } \\
\text { baixos }\end{array}$ & teor baixo & teores médios & teores médios & teores altos & $\begin{array}{l}\text { teores mais } \\
\text { altos }\end{array}$ \\
\hline Mg & teores médios & teor baixo & $\begin{array}{l}\text { teores médios } \\
\text { a médios altos }\end{array}$ & $\begin{array}{l}\text { teores mais } \\
\text { altos }\end{array}$ & teores baixos & $\begin{array}{l}\text { teores muito } \\
\text { baixos a baixos }\end{array}$ \\
\hline A & teor alto & teor altíssimo & teores médios & $\begin{array}{l}\text { teores baixos } \\
\text { a médio-baixos }\end{array}$ & $\begin{array}{l}\text { teores médios } \\
\text { a altos }\end{array}$ & $\begin{array}{l}\text { teores baixos } \\
\text { a médios }\end{array}$ \\
\hline $\mathrm{Fe}$ & teores médios & $\begin{array}{l}\text { teor médio- } \\
\text { alto }\end{array}$ & $\begin{array}{l}\text { teores médios } \\
\text { a baixos }\end{array}$ & $\begin{array}{l}\text { teores baixos } \\
\text { a altos }\end{array}$ & teores altos & teores baixos \\
\hline K & $\begin{array}{l}\text { teores médios } \\
\text { a altos }\end{array}$ & teor médio & $\begin{array}{l}\text { teores médios } \\
\text { e baixos }\end{array}$ & teores baixos & $\begin{array}{l}\text { teores médios } \\
\text { a altos }\end{array}$ & $\begin{array}{l}\text { teores médios } \\
\text { a altos }\end{array}$ \\
\hline
\end{tabular}

Tabela 3 - Teores relativos à mediana para os grupos obtidos pelo programa Isodata.

\begin{tabular}{|c|c|c|c|c|c|c|}
\hline ELEM & GRUPO 1 & GRUPO 2 & GRUPO 3 & GRUPO 4 & GRUPO 5 & GRUPO 6 \\
\hline $\mathrm{Ca}$ & teores baixos & $\begin{array}{l}\text { teores mais } \\
\text { altos }\end{array}$ & teores médios & teores médios & $\begin{array}{l}\text { teores médios } \\
\text { altos }\end{array}$ & $\begin{array}{l}\text { teores mais } \\
\text { baixos }\end{array}$ \\
\hline Mg & teores médios & teores baixos & teores variáveis & teores altos & teores variáveis & $\begin{array}{l}\text { teores médios } \\
\text { baixos }\end{array}$ \\
\hline Al & teores variáveis & $\begin{array}{l}\text { teores baixos } \theta \\
\text { médios baixos }\end{array}$ & teores variáveis & teores variáveis & $\begin{array}{l}\text { teores baixos } \\
\text { a médios }\end{array}$ & $\begin{array}{l}\text { teores } \\
\text { altos }\end{array}$ \\
\hline $\mathrm{Fe}$ & teores variáveis & $\begin{array}{l}\text { teores baixos a } \\
\text { médios-baixos }\end{array}$ & $\begin{array}{l}\text { teores médios } \\
\text { baixos a altos }\end{array}$ & $\begin{array}{l}\text { teores médios } \\
\text { a altos }\end{array}$ & $\begin{array}{l}\text { teores baixos } \\
\text { a médios }\end{array}$ & $\begin{array}{l}\text { teores altos } \\
\text { e muito altos }\end{array}$ \\
\hline K & teores variáveis & $\begin{array}{l}\text { teores baixos a } \\
\text { médios baixos }\end{array}$ & teores baixos & teores variáveis & $\begin{array}{l}\text { teores baixos } \\
\text { médios baixos }\end{array}$ & $\begin{array}{l}\text { teores baixos } \\
\text { a médios }\end{array}$ \\
\hline
\end{tabular}




\section{Comparação dos resultados}

O grupo 1, obtido pelo Isodata, é a soma entre os grupos 1 e 3 obtidos pelo Kmédias, ou seja, é a união entre os dolomitos súticos $\theta$ arenosos da Formação Pedra de Fogo.

O grupo 2, obtido pelo Isodata, é o grupo 6 do K-médias, representam os calcáreos calcfticos da Formaçăo Motuca.

O grupo 3, obtido pelo Isodata, é constituldo de doloesparitos e esparitos areno siltosos da Formação Pedra de Fogo.

Os grupos 4 e 5, obtidos pelo lsodata, compöem o grupo 4 do K-médias, e são constituidos por calcáreos dolomiticos das Formaçōes Pedra de Fogo e Motuca.

O grupo 6, obtido pelo Isodata, é constituido por siltitos micáceos silicosos da Formação Pedra de Fogo. Destaca-se como característica principal os mais baixos teores em Ca.

\section{Conclusão}

Os resultados obtidos pelo K-médias e pelo Isodata mostraram-se muito similares, porém com características distintas. O Isodata separa os grupos ressaltando mais as variáveis com valores e desvios padrões maiores, enquanto no K-médias tal efeito é amenizado.

\section{Varíaveis padronizadas}

Foram realizadas várias tentativas de subdividir em grupos os dados da Tabela 1. Os resultados são apresentados na forma de tabelas, que permitem a distinção das variáveis mais importantes na caracterização de cada grupo.

\section{K-médias (tazendo $K=10$ )}

AMOSTRAS

GRUPO 1: 30A, 30B, 36B, 62C2

GRUPO 2: 41

GRUPO 3: 28F, 31B, 58C 58G, 58H, 62F

GRUPO 4: 35A, 35B, 35Ç 35D

GRUPO 5: 22A, 28A, 28D, 37B, 38B, 62D, 64

GRUPO 6: 28B, 28C 28E, 28G, 31A, 31D, 58A, 90

GRUPO 7: 13, 22B, 38A 
GRUPO 8: 32A, 58B, 89A, 89B

GRUPO 9: 22C 37A, 79

GRUPO 10: 52A, 88, 62C1

Isodata

Adotando-se desvio padrão máximo 26, distância mínima 23 e número mínimo por grupo igual a 2, obtém-se:

Amostras eliminadas: $13,31 \mathrm{~A}, 31 \mathrm{~B}, 58 \mathrm{~B}, 64$

GRUPO 1: 28B, 28C, 28E, 28G, 58A

GRUPO 2: 31D, 38B, 62F, 90

GRUPO 3: 28D, 35C 62D, 79

GRUPO 4: 35A, 35B, 35D, 38A, 41

GRUPO 5: 32A, 52A, 62C1, 88

GRUPO 6: 89A, 89B

GRUPO 7: 22A, 22B, 30A, 37A, 37B

GRUPO 8: 22C 28A, 36B, 58C

GRUPO 9: 28F, $58 \mathrm{H}$

GRUPO 10: 30B, 58G, 62C2

\section{Comparação e conclusão}

Dos resultados obtidos pelos dois métodos aplicados a variáveis normalizadas conclui-se: o grupo 1 obtido pelo Isodata apresenta todas as unidades de dados dentro do grupo 6 do K-médias. Observa-se nas Tabelas 4 e 5 que estes dois grupos apresentam um comportamento bastante similar para os diversos elementos químicos. A maioria destas amostras é descrita petrograficamente.

$\mathrm{O}$ grupo 1 obtido pelo K-médias agrupa as variáveis $30 \mathrm{~B}$ e $62 \mathrm{C} 1$, que também estão agrupadas no grupo 10 obtido pelo Isodata. Estas amostras estão descritas petrograficamente como silexitos arenosos esparticos magnesianos e silexitos arenosos doloesparticos, respectivamente. Como pode ser observado na Tabela 1, os teores estão bastante próximos para a maioria dos elementos analisados ( $\mathrm{P}, \mathrm{B}, \mathrm{Ca}, \mathrm{Fe}, \mathrm{K}, \mathrm{Mg}, \mathrm{Mn}, \mathrm{Zn}, \mathrm{Zr}, \mathrm{Sr}, \mathrm{Ti})$.

As amostras 31D e 90 do grupo 2 do Isodata também estão contidas dentro do grupo 6 do K-médias e vários elementos possuem comportamento similar $(\mathrm{P}, \mathrm{A}, \mathrm{B}, \mathrm{Ba}, \mathrm{V}, \mathrm{Pb}, \mathrm{Ca}, \mathrm{Cr}$, $\mathrm{Ti}, \mathrm{Fe}$, entre outros). Estas amostras são descritas petrograficamente como doloesparitos silticos 
Tabela 4 - Tabela de teores relativos à mediana das variáveis (elementos químicos), dentro dos grupos obtidos pelo programa K-médias.

\begin{tabular}{|c|c|c|c|c|c|c|c|c|c|c|c|}
\hline \multicolumn{12}{|c|}{ Grupo } \\
\hline Teo & & 1 & 2 & 3 & 4 & 5 & 6 & 7 & 8 & 9 & 10 \\
\hline $\begin{array}{l}B \\
A \\
1 \\
x \\
0\end{array}$ & & $\begin{array}{l}\mathrm{Zr}, \mathrm{Pb} \text {, } \\
\mathrm{Ni}, \mathrm{Cr}, \\
\mathrm{Ti}, \mathrm{V}, \\
\mathbf{P}\end{array}$ & $\begin{array}{l}\mathrm{Al}, \mathrm{Ti}, \\
\mathrm{Pb}, \mathrm{Zr}, \\
\mathrm{Cr}, \mathrm{Mg}, \\
\mathrm{Ni}, \mathrm{B}, \mathrm{Zn} \text {, } \\
\mathrm{Fe}, \mathrm{V}\end{array}$ & & $P, A l$ & & & $\mathrm{Mg}$ & & $\mathrm{Ca}$ & \\
\hline $\begin{array}{l}B \\
A \\
1 \\
x \\
0\end{array}$ & $\begin{array}{l}M \\
E \\
D \\
1 \\
0\end{array}$ & $\begin{array}{l}\mathrm{Mg}, \mathrm{Ca} \\
\mathrm{Al}, \mathrm{Sr}\end{array}$ & & $\begin{array}{l}\mathrm{Na}, \mathrm{Pb}, \\
\mathrm{Al}, \mathrm{Fe}, \\
\mathrm{K}\end{array}$ & $\mathrm{Cr}, \mathrm{Mg}$ & $\begin{array}{l}\mathrm{P}, \mathrm{B}, \mathrm{Ca}, \\
\mathrm{Cr}, \mathrm{Mg}\end{array}$ & $\mathrm{Na}$ & $\mathrm{Ca}, \mathrm{Cr}$ & $\begin{array}{l}\mathrm{Zr}, \mathrm{Al} \\
\mathrm{Fe}, \mathrm{K}, \mathrm{Ti}\end{array}$ & & $\begin{array}{l}\mathrm{Zr}, \mathrm{Al}, \\
\mathrm{Ti}\end{array}$ \\
\hline $\begin{array}{l}\text { M } \\
E \\
D \\
1 \\
0\end{array}$ & & $\mathrm{Fe}$ & $P$ & $\begin{array}{l}\mathrm{Cr}, \mathrm{Mg}, \\
\mathrm{Mn}, \mathrm{Zn}, \\
\mathrm{Sr}\end{array}$ & $\begin{array}{l}\mathrm{Zr}, \mathrm{Ba} \\
\mathrm{K}, \mathrm{Pb} \\
\mathrm{Zn}, \mathrm{Ti} \\
\mathrm{V}\end{array}$ & $\begin{array}{l}\mathrm{Mn}, \mathrm{Pb}, \\
\mathrm{V}\end{array}$ & $\begin{array}{l}\mathrm{Al}, \mathrm{Ca}, \\
\mathrm{Pb}, \mathrm{V}, \mathrm{B} \\
\mathrm{K}, \mathrm{Mn}, \mathrm{Zr}\end{array}$ & $\begin{array}{l}\mathrm{Mn}, \mathrm{V} \\
\mathrm{Zn}\end{array}$ & $\begin{array}{l}\mathrm{Ba}, \mathrm{Ca}, \\
\mathrm{Pb}, \mathrm{Zn}\end{array}$ & $\mathrm{P}, \mathrm{Cr}, \mathrm{Fe}$ & $\mathrm{Na}, \mathrm{P}$ \\
\hline $\begin{array}{l}\text { M } \\
\text { É } \\
D \\
1 \\
0\end{array}$ & $\begin{array}{l}A \\
L \\
T \\
0\end{array}$ & & & $\begin{array}{l}\mathrm{B}, \mathrm{Ba} \\
\mathrm{V}\end{array}$ & $\begin{array}{l}\mathrm{B}, \mathrm{Fe}, \mathrm{Mn} \text {, } \\
\mathrm{Sr}\end{array}$ & $\begin{array}{l}\mathrm{Al}, \mathrm{Fe}, \mathrm{K}, \\
\mathrm{Zr}, \mathrm{Ti}\end{array}$ & $\begin{array}{l}\mathrm{Cr}, \mathrm{Fe}, \\
\mathrm{Mg}, \mathrm{Ti}\end{array}$ & $\begin{array}{l}\mathrm{Ba}, \mathrm{Fe} \\
\mathrm{K}, \mathrm{Pb}\end{array}$ & $\mathrm{Mg}, \mathrm{Cr}$ & $\begin{array}{l}\mathrm{Mg}, \mathrm{Pb}, \\
\mathrm{Zr}, \mathrm{Sr}, \\
\mathrm{Ti}, \mathrm{K}\end{array}$ & $P, B$ \\
\hline $\begin{array}{l}A \\
L \\
T \\
0\end{array}$ & & & $\mathrm{Ca}, \mathrm{K}$ & & $\mathrm{Ca}, \mathrm{Na}$ & & & $\begin{array}{l}\mathrm{Al}, \mathrm{Zr}, \\
\mathrm{Ti}\end{array}$ & $\mathrm{Na}, \mathrm{Sr}$ & $\mathrm{B}, \mathrm{Ba}$ & $\begin{array}{l}\mathrm{Ba}, \mathrm{Ca} \\
\mathrm{V}, \mathrm{Cr}, \mathrm{Mg} \\
\mathrm{Zn}, \mathrm{Ni}\end{array}$ \\
\hline $\begin{array}{l}V \\
A \\
R \\
1 \\
0\end{array}$ & & $\begin{array}{l}\mathrm{Mn}, \mathrm{B}, \\
\mathrm{Na}\end{array}$ & & $\begin{array}{l}\mathrm{P}, \mathrm{Zr}, \\
\mathrm{Ca}, \mathrm{Ti}, \\
\mathrm{Ni}\end{array}$ & $\mathrm{Ni}$ & $\begin{array}{l}\mathrm{Ba}, \mathrm{Na}, \\
\mathrm{Zn}, \mathrm{Ni} \text {, } \\
\mathrm{Sr}\end{array}$ & $\begin{array}{l}\mathrm{P}, \mathrm{Ba}, \mathrm{Sr}, \\
\mathrm{Ni}, \mathrm{Sr}\end{array}$ & $\begin{array}{l}\mathrm{P}, \mathrm{B}, \mathrm{Na} \text {, } \\
\mathrm{Ni}, \mathrm{Sr}\end{array}$ & $\begin{array}{l}\mathrm{P}, \mathrm{B}, \mathrm{Ni}, \\
\mathrm{V}\end{array}$ & $\begin{array}{l}\mathrm{Al}, \mathrm{Mn}, \\
\mathrm{Na}, \mathrm{Zn}, \\
\mathrm{V}\end{array}$ & $\begin{array}{l}\mathrm{Fe}, \mathrm{K}, \mathrm{Mn} \\
\mathrm{Sr}\end{array}$ \\
\hline
\end{tabular}


Tabela 5 - Tabela de teores relativos à mediana das variáveis (elementos químicos), dentro de cada grupo obtido pelo programa Isodata.

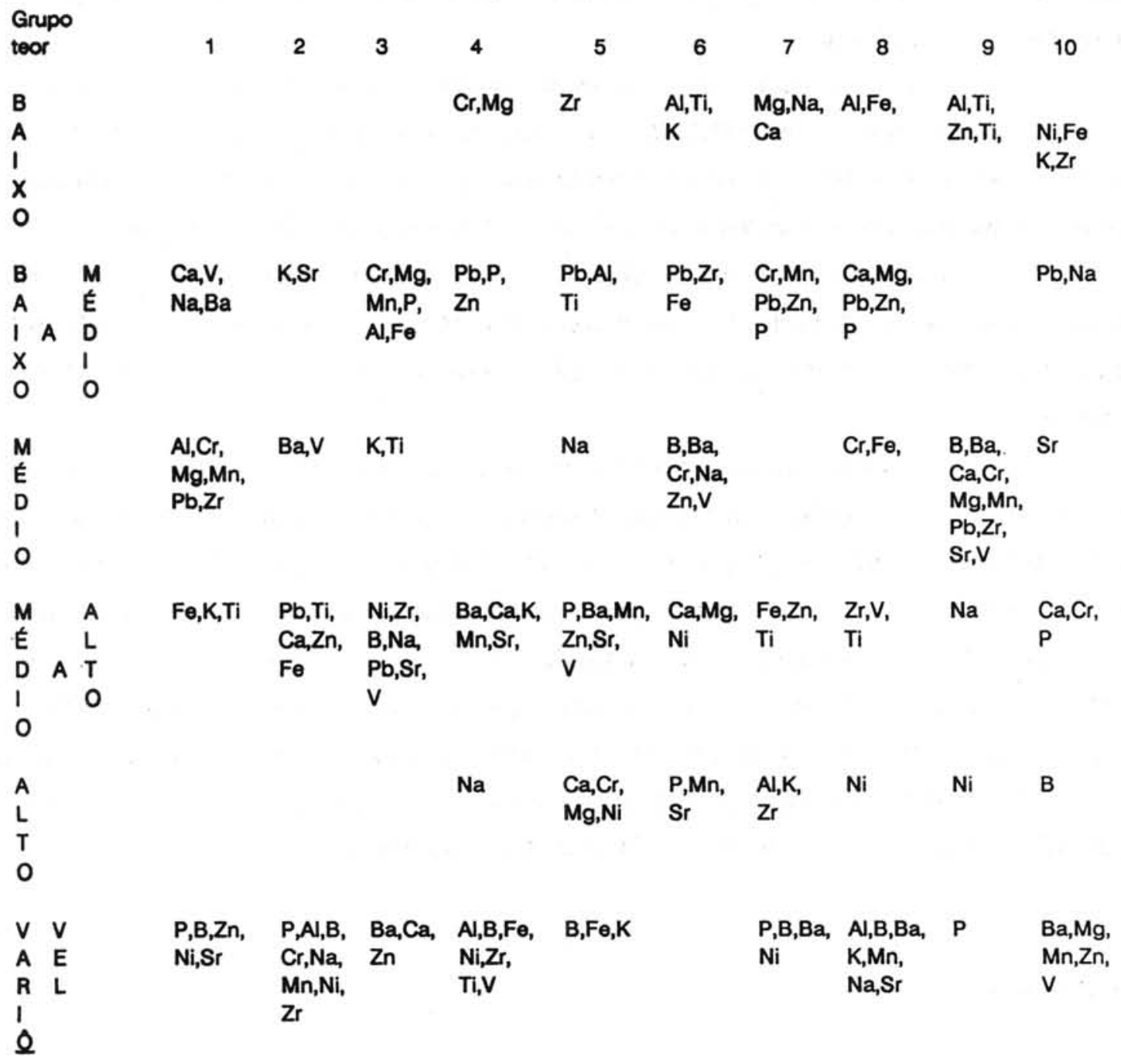


silicosos e doloesparitos silicosos, respectivamente.

O grupo 4 do Isodata também apresenta grande similaridade no comportamento dos teores relativos (Tabelas 4 e 5), com o grupo 4 do $\mathrm{K}$-médias, ambos contendo as amostras 35A, 35B e 35D. No grupo 9 do lsodata e no grupo 3 do K-médias encontram-se as amostras $28 \mathrm{~F}$ e $58 \mathrm{H}$, que nas diversas partições obtidas sempre se apresentaram juntas. Isto geralmente ocorre entre amostras multo próximas. Já o grupo 5 do lsodata apresenta as amostras $52 \mathrm{~A}, 62 \mathrm{C} 1$ e 88 , que formam - grupo 10 do K-médias. Estes grupos apresentam teores relativos bastante similares para quase todos os elementos analisados.

Outras relações podem ser estabelecidas entre o conteúdo dos diversos grupos, porém não nos demoraremos mais estabelecendo estas relações. O leitor pode observar que quando as variáveis são padronizadas, o comportamento dos grupos não sofre mais a influência das variáveis (elementos) com valores mais expressivos, mas de todas as variáveis igualmente.

Até agora fol observado uma série de similaridades entre os grupos formados pelo K-médias e pelo Isodata. No entanto, uma breve análise mostra que existem algumas divergências entre os grupos obtidos por um método em relação aos grupos do outro. $O$ que causa então estas divergências?

Um estudo mais pormenorizado entre os procedimentos destes dois métodos esclarece este comportamento. A diferença básica ocorre porque o Isodata elimina grupos com um pequeno número de unidades de dados. Com isto elimina as grandes distorções que afetam a distribuição interna dos grupos normalmente ocasionadas por um número pequeno de unidades de dados, cujas variáveis possuem valores distantes da maioria dos grupos formados durante o processo. $\mathrm{O}$ método K-médias não elimina grupos, apenas incorpora estas unidades de dados nos grupos existentes. Isto força o deslocamento dos centros dos grupos (dado pelo vetor médio) da região central, para pontos mais periféricos, permitindo a incorporação de novos elementos e ocasionando distorções no comportamento das distribuições internas de cada grupo.

\section{AGRADECIMENTOS}

Agradecemos ao Professor Dr. Armando Márcio Coimbra pela ajuda prestada durante a fase de interpretação dos resultados, ao Professor Dr. Thomas Fairchild pela revisão do abstract e à FAPESP pela bolsa de iniciação cientffica, que permitiu a elaboração deste trabalho. 


\section{REFERÊNCIAS BIBLOGRÁFICAS}

ANDERBERG, M.R. (1973) Cluster analysis for applications. New York, Academyi. Press. 359p.

BAL G.H. \& HAU D.J. (1965) ISODATA, novel method of data analysis and pattern classification. Stanford Res. Inst., Menlo Park, California, Tech. Rep.

BALL, G.H. \& HAL, D.J. (1967) A clustering technique for summarizing multivariate data. Behav. Sci., 12:153-155.

COIMBRA, A.M. (1983) Estudo sedimentológico e qeoquímico do Permo-Tríssico da Bacia do Maranhão. São Paulo. 2v. (Tese de Doutorado - Instituto de Geociências/USP).

COOLEY, W.W. \& LOHNES, P.R. (1971) Multivariate data analysis. New York, John Willey. 364p.

DAVIS, J.C. (1973) Statistics and data analysis in geology. New York, John Willey. 550p.

DIXON, W.J. (1981) BMDP Statistical Software 1981. Berkeley, University of California Press. 727p.

EVERITT, B., (1974) Cluster analysis. London, Heinemann. 122p.

HARALCK, R.M. \& DINSTEIN, I. (1971) An iterative Clustering Procedure. Systems, Man, and Cybernetics, july 1971, v.smc-1, number 3. IEEE Transaction on. New York, N.Y.

MacQUEEN, J.B. (1967) Some methods for classification and analysis of multivariate observations. Proc. Symp. Math. Statist and Probability, 5Th, 1., Berkeley. University of California Press, Berkeley. p.281-297.

TRYON, R.C. \& BAILEY, D.E. (1970) Cluster analysis. New York, McGraw-Hill. 347p. 